\title{
Toward High-Resolution Cryo-Electron Microscopy: Technical Review on Microcrystal-Electron Diffraction
}

\author{
Sangmin Lee ${ }^{\dagger}$, Jeong Min Chung ${ }^{\dagger}$, Hyun Suk Jung* \\ Department of Biochemistry, College of Natural Sciences, Kangwon National University, Chuncheon 24341, Korea
}

\begin{abstract}
${ }^{\dagger}$ These authors contributed equally to this work.

*Correspondence to:

Jung $\mathrm{HS}$,

Tel: +82-33-250-8513

Fax: +82-33-259-5664

E-mail: hsiung@kangwon.ac.kr

crystallization unless the samples are large $(>200 \mathrm{kDa})$ and highly symmetrical. Cryoelectron crystallography is subdivided into the following two categories according to the types of samples: one category that deals with two-dimensional (2D) crystalline arrays and the other category that uses 3D crystals. These two categories of electron-crystallographic techniques use different diffraction data obtained from still diffraction and continuousrotation diffraction. In this paper, we review crystal-based cryo-EM techniques and focus on the recently developed 3D electron-crystallographic technique called microcrystalelectron diffraction.
\end{abstract}

Cryo-electron microscopy (cryo-EM) is arguably the most powerful tool used in structural biology. It is an important analytical technique that is used for gaining insight into the functional and molecular mechanisms of biomolecules involved in several physiological processes. Cryo-EM can be separated into the following three groups according to the analytical purposes and the features of the biological samples: cryo-electron tomography (cryo-ET), cryo-single-particle reconstruction, and cryo-electron crystallography. Cryotomography is a unique EM technique that is used to study intact biomolecular complexes within their original environments; it can provide mechanistic insights that are challenging for other EM-methods. However, the resolution of reconstructed three-dimensional (3D) models generated by cryo-ET is relatively low, while single-particle reconstruction can reproduce biomolecular structures having near-atomic resolution without the need for

Received November 27, 2017

Revised December 10, 2017

Accepted December 10, 2017

Key Words: Cryo-electron microscopy, Cryo-electron tomography, Single-particle analysis, Microcrystal-electron diffraction, High-resolution protein structure

\section{INTRODUCTION}

Cryo-electron microscopy (cryo-EM) is arguably the most powerful and promising tool used in structural biology. It is an important analytical technique that is used for gaining insight into the functional and molecular mechanisms of biomolecules involved in several physiological processes (Dubochet, 2012; Frank, 2016; Henderson, 2004). Cryo-EM can be subdivided into the following three groups according to the analytic purpose and the features of the biological samples: cryo-electron tomography (cryo-ET), cryo-singleparticle reconstruction, and cryo-electron crystallography (Asano et al., 2016; Dubochet, 2012; Frank, 2009; Henderson,
2004; Nannenga \& Gonen, 2014). Cryo-ET is a unique EM technique that is used to study intact biomolecular complexes within their native environments; it can provide mechanistic insights that are challenging for other EM-methods (Asano et al., 2016). However, the resolution of reconstructed threedimensional (3D) models generated by cryo-ET is low, while single-particle reconstruction can reproduce biomolecular structures having near-atomic resolution without the need for crystallization unless the samples are large $(>200$ $\mathrm{kDa}$ ) and highly symmetrical (Frank, 2016). Cryo-electron crystallography is subdivided into the following two categories according to the types of samples: one category that deals with two-dimensional (2D) crystalline arrays (2D

(a) This is an open-access article distributed under the terms of the Creative Commons Attribution Non-Commercial License (http://creativecommons.org/licenses/by-nc/4.0) which permits unrestricted noncommercial use, distribution, and reproduction in any medium, provided the original work is properly cited.

Copyrights @ 2017 by Korean Society of Microscopy 
electron crystallography) and the other category that uses $3 \mathrm{D}$ crystals (3D electron crystallography). These two types of electron-crystallographic techniques use different diffraction data obtained from still diffraction and continuous-rotation diffraction (Nannenga \& Gonen, 2016). In this paper, we review crystal-based cryo-EM techniques and focus on the recently developed 3D electron-crystallographic technique called microcrystal-electron diffraction (micro-ED).

\section{MICROCRYSTAL-ELECTRON DIFFRACTION AND ITS FUTURE DIRECTION}

Since the early 1940s, electron diffraction has been used to study the atomic structures of various materials and biological samples (Bendersky \& Gayle, 2001). The basic principle used in electron crystallography is similar to that of X-ray crystallography, i.e., the crystalline biological samples scatter the incident beam to generate diffraction patterns that can be eventually used for the reconstruction. This technique has been developed over recent years by many groups by using small-sized and relatively dose-insensitive compounds (Nannenga \& Gonen, 2016; Unwin, 2003; Unwin \& Henderson, 1975). In electron crystallography, the crystals should be thinner than those used for X-ray crystallography as electrons have much stronger interactions with the atoms and molecules in the sample than the X-ray photons (Chung et al., 2017; Kimura et al., 1997). Owing to this reason, electron diffraction has been restricted to be used with $2 \mathrm{D}$ crystals that comprise 1 2 thin layers of a well-ordered 2D crystalline lattice. Over the past decades, 2D electron crystallography has been used successfully to obtain near-atomic-resolution structures of membrane proteins surrounded by lipid layers (Gonen et al., 2005; Hyun et al., 2011). However, most structures analyzed by this technique have only moderate resolution (4 10 A) as electrons have extremely high energies and cause large amounts of radiation damage to the crystals, thus resulting in the loss of structural information (Glaeser, 1971). To overcome this loss, the proposed concept has been developed for using hundreds of individual 3D crystals to generate the electron-diffraction patterns, which are then merged into a single dataset. However, until recently, this technique has been unsuccessful in determining the atomic structure of a sample. A study in 2013 introduced a new 3D electron-crystallography technique called micro-ED that provides a striking ability to determine high-resolution protein structures using 3D crystals (Shi et al., 2013).

Micro-ED is the most recently developed cryo-EM technique that allows the collection of high-quality electron-diffraction patterns from extremely small-sized 3D crystals with thicknesses ranging from 0.1 to $0.4 \mathrm{~mm}$ (Shi et al., 2013). Traditional 3D electron crystallography produces a single diffraction pattern for a given $3 \mathrm{D}$ protein crystal owing to the radiation-damage issue. Hence, each diffraction pattern has to be merged into a single dataset to reconstruct the $3 \mathrm{D}$ model; this causes an indexing problem owing to the lack of sufficient information in a single diffraction pattern (Chung et al., 2017). One previous study solved the indexing and merging problems by using a single nanocrystal under low electrondose conditions $\left(\sim 10 \mathrm{e}^{-} / \mathrm{A}\right)$ to collect a complete diffraction dataset (Shi et al., 2013). This initial model of micro-ED used a series of still diffraction patterns (or snapshots) from a single crystal by rotating it through discrete angles among exposures. In this study, a special high-tilt cryo-holder was used to collect the complete tilt-series; it allows the sample to be tilted up to $\pm 70^{\circ}$ in $1^{\circ}$ increments, thus resulting in the production of a $90^{\circ}$ wedge of data from a single crystal. To overcome the "missing wedge" issue, three different crystals were used to produce three sets of diffraction data that were subsequently integrated and merged into one final dataset (Shi et al., 2013). However, the still diffraction method also has the drawback that the rotation must be paused during electronbeam exposure, which leads to a sample-drifting problem. Following this initial proof of the micro-EM principle, an improved data-collection protocol was developed, which is called the continuous-rotation method. This advanced micro-ED data-collection method continuously oscillates the crystal sample during electron-beam exposure, and the diffraction data is recorded using the high frame rate achieved with direct electron detectors (Nannenga et al., 2014b). This improves the data quality by finely sampling reciprocal space (Nannenga et al., 2014b). Because of the many advantages of the continuous-rotation data-collection mode, it has become the standard method for micro-ED, and several highresolution structures have been generated using this technique (Nannenga et al., 2014a; Rodriguez et al., 2015; Yonekura et al., 2015).

With improvements in the detection hardware and imageprocessing software, the fundamental bottlenecks of $3 \mathrm{D}$ crystallography have largely been solved, and several meaningful studies have been reported using the advanced $3 \mathrm{D}$ cryo-EM technique. However, it is still necessary to improve some factors. One of the most interesting current areas of micro-ED is the development of experimental phasing methods because the phasing information that is crucial for crystallography currently cannot be measured from electron-diffraction patterns (Chung et al., 2017). To solve this issue, accurate integrated-diffraction intensities and the development of electron-scattering tables are required (Chung et al., 2017; Nannenga \& Gonen, 2016; Scherer et al., 2014). Several strategies have been used to obtain the phase information, such as the use of heavy atoms for isomorphous replacements and changing the electron wavelengths, but unique and interesting approaches are required to solve the phasing problem (Shi et al., 2016). As the technique is 
further improved and refined, micro-ED appears to be a highly promising technique in cryo-EM, and it seems likely to become a widely used tool for determining the structures of a handful of important biomolecular samples.

\section{CONFLICT OF INTEREST}

No potential conflict of interest relevant to this article was reported.

\section{ACKNOWLEDGMENTS}

This research was supported by Basic Science Research Program through the National Research Foundation of Korea funded by the Ministry of Science, ICT \& Future Planning (NRF-2015R1C1A1A01053611 to H. S Jung), Global Frontier Research Grant (NRF-M1AXA002-2011-0031424 to H. S Jung) and Next-Generation BioGreen Program (SSAC, PJ011891 to H. S Jung).

\section{REFERENCES}

Asano S, Engel B D, and Baumeister W (2016) In situ cryo-electron tomography: A post-reductionist approach to structural biology. J. Mol. Biol. 428, 332-343.

Bendersky L A and Gayle F W (2001) Electron diffraction using transmission electron microscopy. J. Res. Natl. Inst. Stand. Technol. 106, 997-1012.

Chung J M, Lee S, and Jung H S (2017) Recent advances in electron crystallography. Appl. Microsc. 47, 160-164.

Dubochet J (2012) Cryo-EM-the first thirty years. J. Microsc. 245, 221224.

Frank J (2009) Single-particle reconstruction of biological macromolecules in electron microscopy-30 years. Q. Rev. Biophys. 42, 139-158.

Frank J (2016) Generalized single-particle cryo-EM-a historical perspective. Microscopy (Oxf) 65, 3-8.

Glaeser R M (1971) Limitations to significant information in biological electron microscopy as a result of radiation damage. J. Ultrastruct. Res. 36, 466-482.

Gonen T, Cheng Y, Sliz P, Hiroaki Y, Fujiyoshi Y, Harrison S C, and Walz T (2005) Lipid-protein interactions in double-layered two-dimensional AQPO crystals. Nature $\mathbf{4 3 8}$, 633-638.

Henderson R (2004) Realizing the potential of electron cryo-microscopy. Q. Rev. Biophys. 37, 3-13.

Hyun J K, Accurso C, Hijnen M, Schult P, Pettikiriarachchi A, Mitra A K, and Coulibaly F (2011) Membrane remodeling by the double-barrel scaffolding protein of poxvirus. PLoS Pathog. 7, e1002239.

Kimura Y, Vassylyev D G, Miyazawa A, Kidera A, Matsushima M, Mitsuoka K, Murata K, Hirai T, and Fujiyoshi Y (1997) Surface of bacteriorhodopsin revealed by high-resolution electron crystallography. Nature 389, 206-211.

Nannenga B L and Gonen T (2014) Protein structure determination by
MicroED. Curr. Opin. Struct. Biol. 27, 24-31.

Nannenga B L and Gonen T (2016) MicroED opens a new era for biological structure determination. Curr. Opin. Struct. Biol. 40, 128-135.

Nannenga B L, Shi D, Hattne J, Reyes F E, and Gonen T (2014a) Structure of catalase determined by MicroED. Elife 3, e03600.

Nannenga B L, Shi D, Leslie AG W, and Gonen T (2014b) High-resolution structure determination by continuous-rotation data collection in MicroED. Nat. Methods 11, 927-930.

Rodriguez J A, Ivanova M I, Sawaya M R, Cascio D, Reyes F E, Shi D, Sangwan S, Guenther E L, Johnson L M, Zhang M, Jiang L, Arbing M A, Nannenga B L, Hattne J, Whitelegge J, Brewster A S, Messerschmidt M, Boutet S, Sauter N K, Gonen T, and Eisenberg D S (2015) Structure of the toxic core of alpha-synuclein from invisible crystals. Nature 525, 486-490.

Scherer S, Arheit M, Kowal J, Zeng X, and Stahlberg H (2014) Single particle 3D reconstruction for $2 \mathrm{D}$ crystal images of membrane proteins. J. Struct. Biol. 185, 267 277 .

Shi D, Nannenga B L, de la Cruz M J, Liu J, Sawtelle S, Calero G, Reyes F E, Hattne J, and Gonen T (2016) The collection of MicroED data for macromolecular crystallography. Nat. Protoc. 11, 895-904.

Shi D, Nannenga B L, ladanza M G, and Gonen T (2013) Threedimensional electron crystallography of protein microcrystals. Elife $\mathbf{2}$, e01345.

Unwin N (2003) Structure and action of the nicotinic acetylcholine receptor explored by electron microscopy. FEBS Lett. 555, 91-95.

Unwin P N and Henderson R (1975) Molecular structure determination by electron microscopy of unstained crystalline specimens. J. Mol. Biol. 94, 425-440.

Yonekura K, Kato K, Ogasawara M, Tomita M, and Toyoshima C (2015) Electron crystallography of ultrathin 3D protein crystals: atomic model with charges. Proc. Natl. Acad. Sci. USA 112, 3368-3373. 